\title{
LECCIONES DE MECÁNICA DE FLUIDOS EN EL TRATADO HIPOCRÁTICO SOBRE LOS FLATOS
}

\author{
CÉSAR Sierra Martín* \\ cesar.sierra@e-campus.uab.cat
}

\section{RESUMEN}

En el presente trabajo abordaremos el tratado hipocrático Sobre los flatos, conocido por su influencia sofista y vocación retórica. Nuestro objetivo es destacar las analogías y referencias de su autor a leyes y principios físicos como el intercambio de calor, los cambios de estado y la circulación de fluidos. Creemos que todo ello forma parte de la intención de otorgar credibilidad al discurso y a las teorías del autor.

PAlABRAS Clave: Corpus hippocraticum, mecánica de fluidos, física.

\section{Abstract}

This paper is about the Hippocratic treatise On Breaths, characterized by its Sophist influence and its rhetoric vocation. The aim of the article is to highlight the similarities and the references did by the author on physical laws such as heat exchange, phase transition and fluid mechanics. In our opinion this references aimed to reinforce the speech and theories of the author.

KEY WORDS: Corpus hippocraticum, fluid mechanics, physics.

\section{ENTRE SOFISTAS Y MÉDICOS}

El tratado hipocrático Sobre los flatos (=Flat.), escrito entre finales del V y principios del IV a.C., ha suscitado multitud de opiniones entre los 'hipocratistas' modernos, principalmente por dos motivos: la relación de su autor con la sofística y la atribución del contenido del tratado a la escuela de Hipócrates ${ }^{1}$.

* Universitat Autònoma de Barcelona (proyecto RYC2010-05622).

1 Véase la datación de la obra en Segal 1970: 180; Jouanna 1988: 39-49 y López Férez 1988: 27. 
Respecto a la primera cuestión, se acepta que el tratado fue escrito para ser leído en público y que su autor, pese a tener nociones de la medicina de su época, no era médico sino un intelectual que formaba parte de la sofística ${ }^{2}$. Ciertamente, existen motivos para creer que el tratado está dirigido a un público amplio y no muy experto en medicina a te-

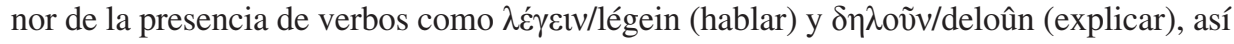
como la abundancia de preguntas retóricas, las sencillas analogías como recurso expositi-

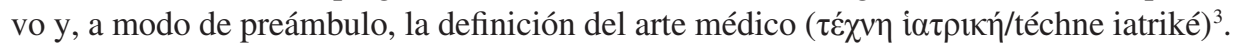
Todo ello está en consonancia con el interés sofista por la medicina como modelo para la retórica y parte fundamental de la educación del ciudadano ${ }^{4}$. Todo ello queda refrendado

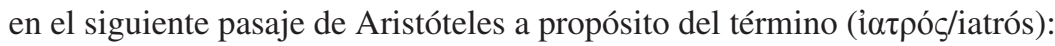

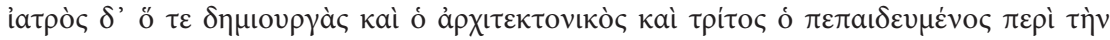

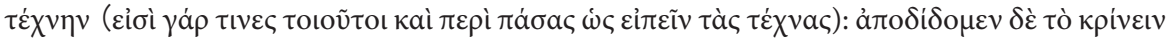

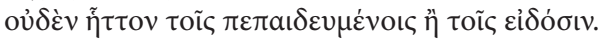

Pero el término médico significa a la vez el practicante ordinario, el que dirige un tratamiento y en tercer lugar el instruido en ese arte. (Tales categorías existen, por así decir, en todas las artes). Y concedemos la facultad de juzgar no menos a los instruidos que a los expertos.

Política $1282 \mathrm{a}^{5}$

Según refiere Aristóteles, en la época clásica existían diferentes categorías dentro de la medicina, que comprendían desde su faceta más práctica a la más teórica. Necesariamente en esta última debían militar muchos intelectuales, griegos bien educados, con amplias nociones en medicina pero sin dedicación expresa. Precisamente, el autor de Flat. constituye un buen ejemplo de este grupo, que los propios médicos entendieron como una amenaza ${ }^{6}$.

Por lo que respecta a su lugar en el $\mathrm{CH}$, el tratado también es conocido por desarrollar ciertos postulados próximos a la escuela pneumática ${ }^{7}$. Sin duda, esta afirmación tiene su fundamento en la suposición de que Hipócrates concedió una extraordinaria importancia al flato ( $\varphi \tilde{v} \sigma \alpha /$ physa) como origen de las enfermedades, algo que aparece reflejado en el Anonymus Londinensis ${ }^{8}$. Esta circunstancia avivó el debate sobre la

${ }^{2}$ Sobre la caracterización del tratado como un discurso epidíctico realizado por un sofista inspirado en Gorgias de Leontinos; véase Jouanna 1984: 28-32; Jouanna 1988: 10-17; López Férez 1988 y Nutton $2004: 50$. Por otro lado, una aproximación a la sofística la tenemos en el clásico de Nestle 2010: 123-160 y en Barney 2009.

3 Jouanna 1984: 29 y López Férez 1988: 42-43.

${ }^{4}$ Relación muy estudiada en nuestros días y que apunta a continuas injerencias de otros sabios en el arte de la medicina; vid. Sobre la medicina antigua 20 (=VM), que delimita claramente las competencias del médico frente a otros sabios de la época, y los siguientes trabajos: Jaeger 1957: 792- 793; Lloyd 1991: 135-138; Longrigg 1993: 93; Jouanna 1999: 82-83; Barton 2005: 41 y ss; Agarwalla 2010: 74 y ss. y Sierra 2012: 94-96.

5 Texto griego en Aristotle. ed. W. D. Ross, Aristotle's Politica. Oxford, Clarendon Press. 1957. Traducción de García Valdés 2000, Gredos.

${ }^{6}$ Nuestro tratado se asemejaría a otros cuyo objetivo no era estrictamente médico, como son: Sobre el médico, Sobre el arte (Laín 1970: 415-417). Para defenderse de estas injerencias externas, el prestigio del médico es fundamental en el Corpus hipocrático (Lara-Nava 2004).

7 Escuela médica post-hipocrática (Nutton 2004: 202 y ss.).

${ }^{8}$ Para hacernos eco de este dato seguimos la edición de Jones 2010: 35. No obstante, el autor de Flat., utiliza de forma muy confusa y a veces contradictoria la terminología referente al aire: $\pi v \varepsilon \tilde{\mu} \mu \alpha /$ pneuma o soplo, 
«cuestión hipocrática», es decir, las auténticas obras de Hipócrates dentro del Corpus hippocraticum ${ }^{9}$. No obstante, la importancia del aire o flato como agente causal de las enfermedades puede rastrearse en la literatura de la época clásica. Tomemos como punto de partida las palabras del propio tratadista:

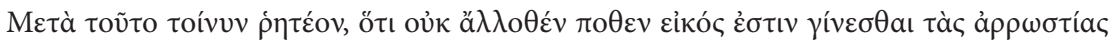

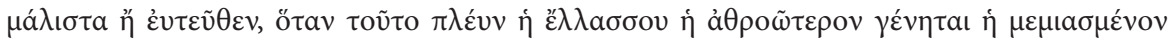

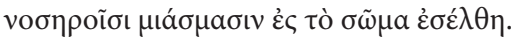

Con que, tras eso, hay que sostener que no cabe esperar que las afecciones se produzcan por ningún otro motivo, sino por el siguiente, a saber, cuando el aire resulta demasiado abundante o escaso, o especialmente compacto; o cuando, infectado de impurezas malsanas, entra en el cuerpo.

Flat. $5^{10}$

La argumentación de que las enfermedades se producían por las impurezas que transportaba el aire enlaza con la idea esgrimida por Tucídides sobre la transmisión de la peste de Atenas ${ }^{11}$. Concretamente, el historiador ateniense sostiene que la epidemia provino de Etiopía, de allí pasó a Egipto, Libia y Oriente Próximo y, finalmente, llegó hasta el Ática (Th. II. 48). Partiendo de esta circunstancia, algunos autores han concluido que existe una relación entre las causas de la enfermedad mostradas por el autor de Flat. y Tucídides ${ }^{12}$. Por tanto, pese a que el testimonio reflejado en Anonymus Londinensis apunte hacia la escuela pneumática, el tema del tratado no parece adelantarse a su época a tenor de los reflejos sobre la contaminación del aire y la peste de Atenas narrados por Tucídides ${ }^{13}$.

Nuestra intención en estas líneas no es profundizar en estos aspectos, sobradamente estudiados en la bibliografía, sino abordar la conexión que intuimos entre el tratadista y la física aplicada ${ }^{14}$. En este sentido, creemos que el tratado presenta argumentos que se alejan de los planteamientos estrictamente médicos y se adentran en el campo de la diná-

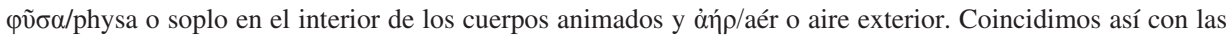
precisiones de López Férez 1988: 34 y, recientemente, Frixione 2012: 6-7.

9 Tema que ha entusiasmado siempre a los 'hipocratistas' y por ello la bibliografía es ingente. Por nuestra parte, proponemos sólo alguna referencia de interés: Lloyd 1975; Edelstein 1987b y Jouanna 1999: 58-71.

${ }^{10}$ Texto griego en Heiberg, J. L., edidit, CMG I 1, Leipzig et Berlin 1927. Traducción de López Férez 1986, Gredos.

${ }^{11}$ En resumen, desde el exterior puede modificarse el equilibrio interno que constituye la salud humana. Esta idea es muy común en el Corpus hipocrático $(=\mathrm{CH})$ y descansa sobre la denominada teoría de los contrarios, que también introduce nuestro autor (Flat. 1), donde las impurezas del aire producen un efecto negativo en el equilibrio interno de las cualidades que configuran la naturaleza humana. Otros ejemplos de esta teoría acerca del aire pueden seguirse en Aër. 2 y Nat.Hom. 9. Véase al respecto Laín 1970: 80; Edelstein 1987a y Thivel 2004.

12 Véanse los motivos en Demont 1983: 341 y 346 y Jouanna 1999: 207-209; y un testimonio similar en Diógenes Laercio, a propósito de una epidemia tratada por Empédocles en Selinunte (D. L. VIII. 70). Además, casi a renglón seguido del argumento en defensa del aire como agente infeccioso, el tratadista define la Peste ( $\lambda$ ourós/loimós) como una fiebre que afecta a un colectivo (Flat. 6), originada también por el aire. Por otro lado, la relación metodológica entre medicina e historia se halla bien definida en Jouanna 2005.

${ }^{13}$ En el mismo Tucídides también son importantes las referencias al aliento fétido, entendido como signo externo observable que la enfermedad producía en el paciente (Th. II. 49. 2). Al respecto, debemos tener presente que la asociación entre el aire exterior y el aliento tiene su reflejo en la cultura médica babilónica (Geller 2006: 188).

${ }^{14}$ Desde el siglo IV a. C., Aristóteles define la mecánica como una aplicación de las matemáticas, es decir, la rama técnica de ésta (Mecánica 847a). 
mica de fluidos y la física. Por consiguiente, descripciones patográficas como el dolor de cabeza (Flat. 8) y la conocida enfermedad sagrada (Flat. 14), adquieren un protagonismo y un cariz especial a la luz de este tratado.

\section{FÍ́SICA Y MEDICINA}

Anteriormente señalábamos que los sofistas estaban instruidos en diversas áreas de conocimiento, entre ellas, la medicina. En consonancia con ello, también había médicos cuya actividad se orientaba hacia contenidos más teóricos e incluso especulativos y que, a su vez, dominaban también otras artes como la retórica, la lógica y la física ${ }^{15}$. Ciertamente,

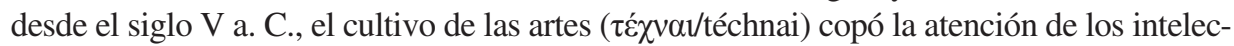
tuales griegos y la mecánica, parte de la física que estudia el movimiento, también formó parte de este proceso ${ }^{16}$. Los primeros testimonios escritos sobre los fenómenos físicos surgen de los filósofos presocráticos, interesados en la investigación de la naturaleza y sus elementos constituyentes. Por ejemplo, desde Anaximandro de Mileto (s. VI. a.C.), encontramos estudios acerca del viento y su relación con la meteorología, así como la justificación de fenómenos como los relámpagos y los truenos a través de los principios físicos del movimiento (fr. 171, 12 A 23, Sén. C.N. II. 18). También son interesantes las precisiones del filósofo milesio sobre el transporte de materia ligera a través de las corrientes de aire, recurso teórico recurrente en el autor de nuestro tratado. Sin embargo, fue Anaxímenes, discípulo de Anaximandro, quien situó al aire y su movimiento como principio y elemento rector del cosmos (fr. 192, 13 A 4, Arist. Met. 984a; fr. 198, Simpl. Fís. 36. 8-13).

Todos estos testimonios y debates sobre la definición de los elementos primordiales dibujan el contexto intelectual de las posteriores investigaciones filosóficas y médicas. En medicina, la teoría de los contrarios adquirió una importancia capital pues sirvió de base teórica para multitud de tratados hipocráticos. Tal teoría introduce la idea de pares de fuerzas opuestas (atracción y repulsión), y tiene en Empédocles de Agrigento a uno de sus principales exponentes (s. V a.C.) ${ }^{17}$. Concretamente, Empédocles refiere que cuatro son los elementos que componen la materia: tierra, aire, agua y fuego, y dos las fuerzas rectoras: Amistad-atracción y Odio-repulsión ${ }^{18}$. Según este esquema, los seres vivos y

15 Son médicos cercanos a la filosofía (van der Eijk 2005: 123), alabados posteriormente por Galeno, El mejor médico es también filósofo (Temkin 1991: 47 y ss.).

${ }^{16}$ La mecánica se desarrolló en contextos técnicos y militares a partir del IV a.C. Véase Gille 1980: 1011; Rihll 1999: 24 y ss.; Clagett 2001: 64 y ss.; y, para la relación entre mecánica y poliorcética, véase De Gandt 2000: 371-378. De hecho, encontramos antecedentes en la filosofía presocrática que tratan de ofrecer una explicación al movimiento de los cuerpos como Parménides y Zenón de Elea o Empédocles de Agrigento (Irby-Massie; Keyser 2002: 150-151.). No obstante, el término mecánica se expandió profusamente en la cultura griega con autores como Aristóteles, Mecánica, que trata sobre el diseño de ingenios y la aplicación técnica de las leyes de la física; y que tendrá una gran influencia en el Renacimiento (De Gandt 2000: 375). Así, la mecánica alcanzaría su máxima expresión siglos después con figuras como las de Vitruvio (s. I a.C.) y Herón de Alejandría (s. I d. C.). Sobre esta cuestión véase Gille 1980: 122-145 y Laird-Roux 2007: 3.

17 Véase al respecto Sambursky 1960: 18-19. La teoría de los contrarios puede advertirse también en Anaximandro (fr. 128, 12 A 9, Simpl. Fís. 24. 13-25) y en Alcmeón de Crotona (Aëtius, V 30, 1; D.K.24B4), véase al respecto Edelstein 1987c: 351-354; Longrigg 1993: 47-51 y Sierra 2012: 91-93. Pero es Empédocles quien constituye el eslabón entre la magia y medicina pragmática (Laín 2005: 83-86) y referente inmediato para algunos tratadistas hipocráticos (Lloyd 1999: 35 y ss.; Barton 2005: 30 y la relación con VM 20).

18 Véanse por ejemplo: fr. 313 (31 B 6, Aecio I. 3. 20); fr. 317 (31 B 26, Simpl., Fís. 33. 18) y fr. 318 (31 A 28, Arist. Met. 984a). Editados y traducidos al castellano en la colección Gredos. 
el resto de cuerpos que configuran la Tierra surgen por combinación de estos elementos bajo la acción de dichas fuerzas ${ }^{19}$.

En muchos aspectos, el tratado que nos ocupa adapta los anteriores razonamientos filosóficos al estudio del cuerpo humano, es decir, pasa del macrocosmos (universo) al microcosmos (interior de los cuerpos vivos), proceso frecuente en el $\mathrm{CH}$ (Flat. 3) ${ }^{20}$. Según nuestra impresión, al abordar el microcosmos en el CH surgen, como mínimo, dos problemas: la diversidad de teorías sobre la naturaleza del hombre y el movimiento que generan los distintos fluidos en el interior del cuerpo. Ambos problemas están relacionados y responden a sendas problemáticas en la interpretación del macrocosmos. Así, de la misma forma que había diferentes teorías acerca de la composición de los elementos primordiales del universo, también existían diversas teorías acerca de las sustancias constituyentes del cuerpo humano $^{21}$. En consecuencia, la idea que el médico poseía sobre las sustancias o fluidos que componían el cuerpo humano repercutía en su concepción del movimiento de dichos flujos internos ${ }^{22}$. Al respecto, el autor de Flat., ofrece una buena muestra de su idea acerca de la fisiología humana y la dinámica de fluidos interna en el siguiente pasaje a propósito del origen de los escalofríos:

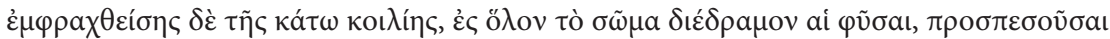

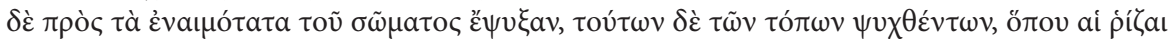



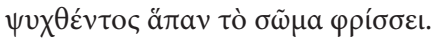

Obstruida la cavidad inferior, los flatos se extienden por todo el cuerpo y, cuando caen en las partes más sanguíneas del cuerpo, las enfrían. Una vez fríos estos lugares, donde están las raíces y fuentes de sangre, un escalofrío corre por todo el cuerpo, y, cuando toda la sangre se ha enfriado, todo el cuerpo siente escalofríos.

Flat. 7. 14-18

En este pasaje, el autor del tratado entiende el interior del ser humano como una mezcla de aire y sangre aunque no se aprecia una postura clara sobre la constitución del hombre ${ }^{23}$.

19 Además otorga al aire la capacidad de insuflar vida (fr. 313, vid. supra), lo cual apunta a una relación con los postulados médicos a los que hacíamos referencia anteriormente (Jouanna 1988: 25-29). Por su parte Thivel (2005: 240-241), analiza la evolución conceptual del aire como elemento patogénico, distinguiendo tres etapas: la arcaica (escritos homéricos), la «empedoclea», que llega hasta la época de Platón, y la aristotélica.

${ }^{20}$ Abstracción planteada por Demócrito (DK 68 B 34), que trata de establecer los principios rectores del universo como modelo para interpretar el cuerpo humano (Le Blay 2005).

${ }^{21}$ Principalmente pueden distinguirse tres teorías sobre la naturaleza humana en la medicina hipocrática: la humoral, que seguía la teoría de los 4 humores (bilis negra, bilis amarilla, sangre y pituita), la elemental, seguidora de la doctrina de los 4 elementos de Empédocles (aire, agua, tierra y fuego), y la dinámica, centrada en 4 cualidades contrapuestas: caliente, frío, seco y húmedo (Laín 1982: 14). No obstante, estas tres teorías no dejan de ser una pequeña muestra de lo que debió constituir un vivo debate en el seno de la medicina hipocrática y la filosofía, vid. Pigeaud 1996: 778-780.

${ }_{22}$ Cuestión propia de la medicina interna y tema transversal en el $\mathrm{CH}$, principalmente: Lugares en el hombre, Carnes, Glándulas, Epidemias y Enfermedades. Sin duda, una especialista en la materia es Elizabeth Craik, quien toma como referencia el tratado Glándulas y distingue hasta siete rutas del flujo interior: orejas, ojos y nariz, establecen sendas rutas con la cabeza; la espalda y la cadera, reciben flujo a través de los vasos o venas y, por último, las rutas que van del paladar a los pulmones y de aquel al vientre (Craik 2001: 105 y Craik 2009: 22 y ss.). La misma autora realiza un análisis análogo para el tratado Lugares en el hombre (Craik 1999).

${ }^{23}$ En Flat. 10 aparecen otros humores como la flema, lo cual evidencia la confusión del tratadista en esta 
No obstante, pese a que el autor no parece tener muy claro el esquema fisiológico sobre el que quiere disertar, sí apreciamos la voluntad de clarificar ciertos fenómenos (escalofríos, bostezos, eructos...), utilizando analogías que hacen referencia a principios físicos. En nuestro caso, el escalofrío se produce por enfriamiento de la sangre, esto es, por un intercambio de calor entre el aire, que entra por ingestión junto a los alimentos, y la sangre ${ }^{24}$. También aplicando principios mecánicos se intenta explicar el origen de los dolores de cabeza:

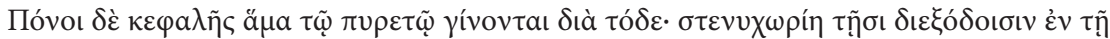

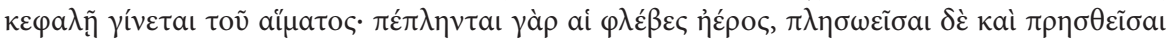



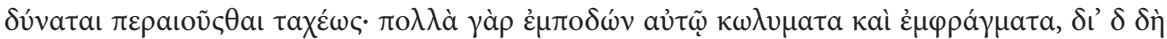

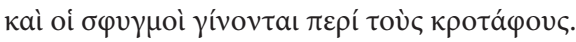

Se producen dolores de cabeza al mismo tiempo que la fiebre por la siguiente razón: se les forma una estrechez a los pasos que la sangre tiene en la cabeza, pues las venas están llenas de aire, y, al estar llenas e inflamadas, causan dolor de cabeza. Efectivamente, la sangre, obligada por la fuerza a meterse por caminos estrechos, como está caliente, no puede pasar con rapidez, porque tropieza con muchos obstáculos e impedimentos. Por esta causa, se producen los latidos en las sienes.

Flat. 8

En los dos últimos pasajes, el autor de Flat. plantea dos de sus recursos maestros para explicar el origen de las dolencias: el intercambio de calor entre el aire y el interior del cuerpo, que incluso puede causar cambios de estado en los fluidos internos; y la circulación de la mezcla heterogénea aire-sangre, que depende de la tortuosidad del camino. Estas ideas podrían resultar familiares al público del tratadista pues la dinámica de fluidos en la cultura griega se asociaba fundamentalmente a la navegación marítima ${ }^{25}$. En este sentido, las leyes y principios físicos que podían recordar al gobierno de una nave: accidentalidad del camino, velocidad de las corrientes marinas, dirección del viento, etc, podían ser argumentos asequibles para el auditorio. Análogamente, Aristóteles Mecánica 848a, afirma que los artesanos utilizan los principios matemáticos y físicos del círculo para fabricar artilugios y los marineros reman gracias a la ley de la palanca (Mecánica 850b). En ambos casos emplean la mecánica aún sin conocerla en profundidad, lo cual introduce al público en una atmósfera familiar que el autor utiliza para generar empatía y verosimilitud a sus palabras. Sobre este último punto trata el anterior pasaje pues, según lo entendemos, el aire hace aumentar el volumen de los conductos por los que pasa la sangre (venas) y ello conlleva que la circulación por los $\operatorname{pasos}^{26}$ ( $\pi$ ópo//póroi) de la cabeza se haga más difícil y lenta, produciendo el dolor de cabeza.

materia. Como anota López Férez 1988: 39, el autor tampoco define exactamente la manera en que la sangre llega a calentarse después (Flat. 8. 26).

${ }^{24}$ Análogamente describe los temblores, que son el resultado de los escalofríos (Flat. 8). Coincidimos con López Férez 1988: 39, al señalar que la terminología que utiliza el autor para describir el movimiento de la sangre, utilizando el verbo ( $\kappa \alpha \theta \dot{\alpha} \lambda \lambda$ o $\mu \alpha 1 /$ kathálomai) es muy extraña en los tratados médicos griegos. Por otro lado, las interacciones entre el calor y el aire en el CH han sido abordadas por Frixione 2012: 13-18.

25 Sobre la dinámica de fluidos y la navegación en el mundo greco-romano, véase Frau 1987: 206-215.

${ }^{26}$ En absoluto resulta extraño hablar de «pasos» por donde circulan los flujos, «recipientes» en vez de órganos, etc., lo cual plantea no pocos problemas de traducción en el $\mathrm{CH}$. Al respecto véase el interesante análisis del tratado Sobre la anatomía en E. Craik (Craik 1998) y M. P. Duminil 1998 y las precisiones sobre la interpretación hipocrática de los conductos por donde circula la sangre en Kollesch 2007. En conjunto, es imprescindible partir de la obra de Duminil 1983. 
Centrándonos en la intención del autor, creemos que el tratadista busca ganar la aprobación del público, presentando una respuesta a un fenómeno común (el dolor de cabeza) mediante una analogía fundamentada en la mecánica de fluidos. Dicho de otra forma, el autor considera que la cantidad de obstáculos que la mezcla aire-sangre encuentra en su recorrido por la cabeza son el origen del dolor, por acumulación de sangre, y ofrece el latido de las sienes como prueba palpable de ello ${ }^{27}$.

A modo de colofón, el autor nos ofrece una explicación de cómo se origina la denominada enfermedad $\operatorname{sagrada}^{28}$ (Flat. 14). A grandes rasgos, el autor entiende la enfermedad sagrada como una privación de la inteligencia y ésta, según su propia teoría, dependía en grado sumo de la sangre ${ }^{29}$. Pues bien, aplicando el mismo concepto que ya utilizara para el dolor de cabeza, el tratadista afirma que un exceso de aire dificulta, por el peso y la presión que ejerce, el paso de la sangre en la cabeza, generando una corriente sanguínea irregular y trastornos psíquicos observables desde el exterior. Por tanto, estamos de nuevo ante una argumentación cuyos cimientos apuntan hacia la dinámica de fluidos. Todavía más, el autor presenta otra explicación física sobre uno de estos signos externos, la espuma blanca:

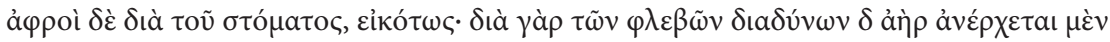

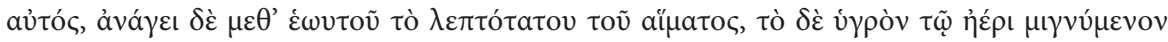

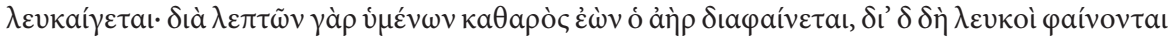
$\pi \alpha v \tau \varepsilon \lambda \tilde{\omega} \varsigma$ oi ả $\varphi \rho \rho o ́$.

La espuma corre por la boca, como cabe esperar, pues el aire metiéndose por las venas, sube por sí mismo, pero lleva consigo la parte más fina de la sangre. Y el líquido, al mezclarse con el aire, se vuelve blanco, porque el aire, cuando está puro, se deja ver a través de finas membranas. Por ello, la espuma parece totalmente blanca.

Flat. 14

Por un lado, el pasaje muestra nuevamente la imprecisión y confusión del autor en materia fisiológica pero, por otro, nos remite a los postulados físicos. Esta vez, el autor plantea que el aire interno, compuesto más volátil que la sangre, abandona el cuerpo debido a su exceso, arrastrando la fracción más ligera de la sangre y mezclándose para adquirir una coloración característica. Por tanto, el proceso de separación físico (parte más ligera de la sangre) y la posterior mezcla, se ponen de nuevo al servicio de la argumentación de un fenómeno observable.

Los argumentos acerca de la enfermedad sagrada encuentran paralelos en el tratado Morb.Sacr., que sitúa el origen de las enfermedades psíquicas en el cerebro ${ }^{30}$

27 Aunque el autor yerre en la explicación y ésta sea incluso incoherente, analizamos la intencionalidad que parece desprenderse de las continuas analogías y referencias a principios físicos: bien sea intercambio de calor o dinámica de fluidos.

${ }^{28}$ La enfermedad sagrada era un tema sobre el que existía un gran debate en la época clásica puesto que, aparte del caso que nos ocupa, tenemos también el tratado Sobre la enfermedad sagrada (= Morb.Sacr.), alguna referencia en Aristóteles, De somno et vigilia, y hasta un pasaje en Heródoto (III. 33). Véase Dodds 1980: 72 y, especialmente, Temkin 1994 y van der Eijk 2005: 131-135

${ }^{29}$ El tratadista adopta un punto de vista hematocéntrico respecto a la localización de las cualidades psíquicas o mentales, como la inteligencia. Al respecto, otros tratados enfatizan el papel de la cabeza (encefalocentrismo), Morb. Sacr. 17, o el corazón (cordiocentrismo), Enfermedades y Sobre el corazón. Véase van der Eijk 2005: 124-125.

${ }^{30}$ Al respecto véase el análisis completo en Jouanna 1988: 34-37 y la posible influencia del tratadista en posteriores precisiones de Lucrecio sobre la enfermedad sagrada (Segal 1970). 
(Morb.Sacr. 17). En concreto, se apunta a que la acumulación de flema y bilis es el origen de la locura e incluso se defiende la función reguladora térmica de la sangre (Mob.Sacr. 18). En este sentido, la acumulación de sangre en la cabeza produce un aumento de la temperatura, alterando el estado natural de los humores y produciendo trastornos mentales. A su vez, el mismo tratadista también resalta el importante papel del aire en este proceso, otorgándole la capacidad de infundir entendimiento al ser humano (Morb.Sacr. 19). Por este motivo, el autor argumenta que el aire circula por el cuerpo comenzando por la cabeza, para aprovecharlo en su estado más puro, y luego se distribuye por el resto del cuerpo ${ }^{31}$. Según nuestra opinión, el autor de Morb.Sacr. no otorga la misma relevancia ni el mismo grado de detalle a los procesos físicos y la dinámica de fluidos que, supuestamente, originaban los síntomas de la enfermedad sagrada en Fat., puesto que no se realizan las analogías que apuntan hacia la dinámica de fluidos (tortuosidad y otros problemas de circulación) y los fenómenos térmicos (dilatación de los conductos internos).

\section{ARgumentos Físicos PARA PROBLEMAS MÉdicos}

En concusión, no contravenimos la idea comúnmente aceptada de que el autor del tratado Sobre los flatos fuera en realidad un sofista. Más bien rubricamos esa idea pues su lectura genera la impresión de que el autor no era un experto en medicina ${ }^{32}$. No obstante, entendemos que el tratado llama la atención por las abundantes referencias a principios físicos tales como el intercambio de calor, los cambios de estado y la circulación de fluidos. El tratadista, amparado en la física, trata de fundamentar sus teorías acerca del origen de ciertos males como el dolor de cabeza (Flat. 8), la hidropesía (Flat. 12) y la enfermedad sagrada (Flat. 14). En todos los ejemplos anteriores el aire y la sangre juegan un papel determinante y se describen con las mismas leyes físicas que rigen el exterior (del macrocosmos al microcosmos). Por otro lado, tampoco concebimos al autor como un especialista en dinámica pues no alcanza la complejidad de Aristóteles Mecánica o Euclides Elementos, e incurre en las mismas imprecisiones y confusiones, que dan pie a señalar que no se trata de un médico. Así pues, creemos que los conocimientos en física del autor se enmarcan también dentro del amplio interés que la sofística mostró por gran variedad de artes y disciplinas del saber. Estamos pues ante un discurso epidíctico en el que, al parecer, los argumentos cimentados en la física gozaban de credibilidad ante el auditorio. Dicho de otro modo, el autor entendía que la analogía mediante la física otorgaba verosimilitud a sus teorías sobre el origen de las enfermedades y el funcionamiento interno del cuerpo.

\section{Texto Aristóteles:}

Texto griego: Ross, W.D. (1957), Aristotle's Politica. Oxford: Clarendon Press. 1957. Traducción: García ValdÉs, M. (2000), Aristóteles. Política, Madrid: Gredos.

31 De nuevo la circulación interna de los fluidos no queda clarificada fisiológicamente. Sobre la interpretación del movimiento del aire en el interior del cuerpo en el tratado Morb.Sacr. véase Frixione 2012: 15-16.

${ }^{32}$ Remitimos a los argumentos de López Férez 1988. 
Textos Sobre los flatos:

Texto griego: Heiberg, J.L. (1927), CMG I. 1.

Traducción: López Férez, J.A. (1986), Tratados hipocráticos II, Madrid: Gredos.

\section{REFERENCIAS BIBLIOGRÁFICAS}

Agarwalla, P. (2010), «Training Showmanship. Rhetoric in Greek medical education of the fifth and fourth centuries BC» en, Horstmanshoff, M. (ed.), Hippocrates on medical education. Selected papers read at the XIIth international Hippocrates Colloquium, Universiteit Leiden 24-26 August 2005, Leiden: Brill: 73-85.

Barney, R. (2009), «The Sophistic movement» en, Gill, M. L.; Pellegrin, P. (eds.), A Companion to ancient philosophy, Malden: Blackwell: 77-97.

Barton, J. (2005), «Hippocratic explanations» en, Van der Eijk, Ph. (ed), Hippocrates in Context. Papers read at the XIth International Hippocrates Colloquium. University of Newcastle Upon Tyne. 27-31 August 2002, Leiden-Boston: Brill: 29-47.

Clagett, M. (2001), Greek Science in Antiquity, New York: Abelard-Schuman. (1 $1^{\text {a }}$ edición 1955, New York).

Craik, E. (1998), «The Hippocratic treatise on Anatomy», CQ 48 (1): 135-167.

- (1999), «Places in man: flux theory and therapeutic intervention» en, Garofalo, I; Lami, A.; Manetti, D.; Roselli, A. (eds.), Aspetti della terapia nel Corpus Hippocraticum. Atti del IX $\mathrm{X}^{\mathrm{e}}$ Colloque International Hippocratique. Pisa 25-29 settembre 1996, Firenze: Leo S. Olschki: 177-182.

- (2001), «Thucydides on the plague: physiology of flux and fixation», CQ 51(1): 102-108.

- (2009), The Hippocratic treatise 'On Glands', Leiden: Brill.

De Gandt, F. (2000), «Tecnología» en, Brunschwig, J; Lloyd, G. E. R. (eds.), El Saber griego: diccionario crítico, Madrid: Akal: 371-378. (1ª edición 1996, Paris).

Demont, P. (1983), «Notes sur le récit de la pestilence athénienne chez Thucydide et sur ses rapports avec la médicine grecque de l'époque classique» en: Lasserre, F. (ed), Formes de pensée dans la Collection hippocratique. Actes du IVe colloque international hippocratique, Lausanne, 21-26 septembre 1981, Gèneve: Droz: 341-353.

Dodds, E. R. (1980), Los griegos y lo irracional, Madrid: Alianza. (1ª edición 1951, Berkeley).

Duminil, M. P. (1983), Le sang, les vaisseaux, le cœur: dans la Collection hippocratique : anatomie et physiologie, Paris: Les Belles Lettres.

- (1998), «Notice» en, Hippocrate. Plaies, Nature des Os, Cour, Anatomie, Paris : Les Belles Lettres (CUF): 199-206.

Edelstein, L. (1987a), «The Dietetics of Antiquity» en, Temkin, O y Temkin, L. (eds.), Ancient medicine. Selected papers of Ludwig Edelstein, Baltimore: The Johns Hopkins University Press: 303-318. (1ºdición 1967, Baltimore).

- (1987b), «The Genuine works of Hippocrates» en, Temkin, O y Temkin, L. (eds.), Ancient medicine. Selected papers of Ludwig Edelstein, Baltimore: The Johns Hopkins University Press: 133-144. (1º edición 1967, Baltimore).

- (1987c), «Ancient philosophy and medicine», Temkin, O y Temkin, L. (eds.), Ancient medicine. Selected papers of Ludwig Edelstein, Baltimore: The Johns Hopkins University Press: 349-366. ( $1^{\circ}$ edición 1967, Baltimore).

VAN DER EIJK, Ph. (2005), Medicine and philosophy in classical antiquity. Doctors and philosophers on nature, soul, health and disease, New York: Cambridge University Press.

FraU, B. (1987), Tecnologia greca e romana, Gruppo archeologico romano.

Frixione, E. (2012), «Pneuma-Fire Interactions in Hippocratic Physiology», J Hist Med Allied Sci (en prensa). 
Geller, M. J. (2006), «Phlegm and breath- Babylonian contributions to Hippocratic medicine» en, Finkel, I. L.; Geller, M. J. (eds.), Disease in Babylonia, Leiden: Brill: 187-199.

Gille, B. (1980), Les mécaniciens grecs. La naissance de la technologie, Paris: Seuil.

Irby-Massie, G. L.; Keyser, P. T. (2002), Greek Science of the Hellenistic Era, London-New York: Routledge.

JAeger, W. (1957), Paideia: los ideales de la cultura griega, México: fondo de cultura económico. ( $1^{\text {a }}$ edición 1933 , Berlin).

Jones, W. H. S. (2010), The medical writings of Anonymus Londinensis, New York: Cambridge University Press. (1. ${ }^{\mathrm{a}}$ edición 1947, New York).

Jounnna, J. (1984), «Rhétorique et Médecine dans la Collection Hippocratique. Contribution à l'Histoire de la Rhétorique au Ve Siècle», REG 97: 26-44.

- (1988), «Notice» en, Hippocrate. Des Vents-De l'Art, Paris: Les Belles Lettres (CUF): 9-101.

- (1999), Hippocrates, Baltimore : The Johns Hopkins University Press.

- (2005), «Cause and crisis in historical and medical writers of the classical period» en, Van der Eijk, Ph. (ed.), Hippocrates in Context. Papers read at the XIth International Hippocrates Colloquium. University of Newcastle Upon Tyne. 27-31 August 2002. Leiden: Brill: 3-28.

Kollesch, J. (2007), «Phantasie statt Anatomie» en, Boudon-Millot, V.; Guardasole, A.; Magdelaine, C. (eds.), La Science médicale antique. Noveaux regards. Études réunies en l'honneur de Jacques Jouanna, Paris: Beauchesne: 289-293.

Laín Entralgo, P. (1970), La medicina hipocrática, Madrid: Revista de Occidente.

- (1982), El diagnóstico médico. Historia y teoría, Barcelona: Salvat.

- (2005), La curación por la palabra en la Antigüedad clásica, Barcelona: Anthropos. (1ª edición 1958, Madrid).

LAIRD, W. R.; Roux, S. (2007), «Introduction» en, Laird, W. R.; Roux, S. (eds.), Mechanics and natural philosophy before the scientific revolution, Dordrech: Springer: 1-11.

Lara Nava, M. D. (2004), «El prestigio del médico hipocrático», $C F C(g)$ 14: 45-58.

LE BLAY, F. (2005), «Microcosm and macrocosm: the dual direction of analogy in Hippocratic thought and the meteorological tradition», en Van der Eijk, Ph. (ed.), Hippocrates in Context. Papers read at the XIth International Hippocrates Colloquium. University of Newcastle Upon Tyne. 27-31 August 2002. Leiden-Boston: Brill: 251-270.

- (2005), «Air; pneuma and breathing from Homer to Hippocrates» en Van der Eijk, Ph. (ed.), Hippocrates in Context. Papers read at the XIth International Hippocrates Colloquium. University of Newcastle Upon Tyne. 27-31 August 2002. Leiden-Boston: 73-85.

Lloyd, G. E. R. (1975), «The Hippocratic Question», CQ 25 (2): 256-260.

- (1991), «The social background of early greek philosophy and science» en, Methods and Problems in Greek Science. Selected papers. Cambridge: Cambridge University Press: 121-140.

- (1999), Magic, reason and experience. Studies in the origins and development of greek science, London: Duckworth. ( $1^{\circ}$ edición 1979, Cambridge).

Longrigg, J. (1993), Greek rational medicine. Philosophy and medicine from Alcmaeon to the Alexandrians, London-New York: Routledge.

LóPEZ FÉrez, J. A. (1988), «Sobre los flatos» como reflejo de la sofística en el Corpus hippocraticum», CFC 21: 25-43.

Nestle, W. (2010), Historia del espíritu griego, Barcelona: Ariel. (1ª edición 1944, Stuttgart).

Nutton, V. (2004), Ancient medicine, London: Routledge.

Pigeaud, J. (1996), «Il medico e la malattia» en, Settis, S. (ed.), I Greci. Storia, Cultura, Arte e Società, v. 1, Torino: Einaudi: 771-814.

Rinll, T. E. (1999), Greek Science, Oxford: Oxford University Press.

Sambursky, S. (1960), The Physical world of the Greeks, London: Routledge. (1 a edición 1956, London). 
Segal, Ch. (1970), «Lucretius, Epilepsy, and the Hippocratic on Breaths», CPh 65(3): 180-182.

Sierra, C. (2012), «Notas sobre medicina y difusión de ideas en la Grecia clásica», $C F C(g)$ 22: 91-101.

Temkin, O. (1991), Hippocrates in a world of pagans and Christians, Baltimore: The Johns Hopkins University Press.

- (1994), The falling sickness: a history of epilepsy from the Greeks to the beginnings of modern neurology, Baltimore: The Johns Hopkins University Press. (1 $1^{\text {a }}$ edición 1945, Baltimore).

Thivel, A. (2004), «Eryximaque et le principe des contraires», $C F C$ ( $g$ ) 14:35-44.

- (2005), «Air, pneuma and breathing from Homer to Hippocrates» en Van der Eijk, Ph. (ed.), Hippocrates in Context. Papers read at the XIth International Hippocrates Colloquium. University of Newcastle Upon Tyne. 27-31 August 2002. Leiden-Boston: Brill: 73-85. 